\title{
The safety of Pipeline flow diversion in fusiform vertebrobasilar aneurysms: a consecutive case series with longer-term follow-up from a single US center
}

\author{
Sabareesh K. Natarajan, MD, MS, ${ }^{1,2}$ Ning Lin, MD, ${ }^{1-3}$ Ashish Sonig, MD, MS, MCh, ${ }^{1,2}$ \\ Ansaar T. Rai, MD, ${ }^{4}$ Jeffrey S. Carpenter, MD, ${ }^{4}$ Elad I. Levy, MD, MBA, ${ }^{1,2,5,6}$ and \\ Adnan H. Siddiqui, MD, $\mathrm{PhD}^{1,2,5-7}$
}

\begin{abstract}
1Department of Neurosurgery, Jacobs School of Medicine and Biomedical Sciences, University at Buffalo, State University of New York, Buffalo; '2Department of Neurosurgery, Gates Vascular Institute/Kaleida Health, Buffalo; ${ }^{3}$ Department of Neurosurgery, Weill Cornell Medical College, New York City, New York; ${ }^{4}$ Department of Radiology, West Virginia University, Morgantown, West Virginia; ${ }^{5}$ Department of Radiology, School of Medicine and Biomedical Sciences, University at Buffalo, State University of New York; ${ }^{6}$ Toshiba Stroke and Vascular Research Center, University at Buffalo, State University of New York, Buffalo; and ${ }^{7}$ Jacobs Institute, Buffalo, New York
\end{abstract}

OBJECTIVE Pessimism exists regarding flow diversion for posterior circulation aneurysms because of reports of perforator territory infarcts and delayed ruptures. The authors report the results of patients who underwent Pipeline Embolization Device (PED) flow diversion using novel strategies for treatment of fusiform posterior circulation aneurysms, and compare these results with those from previously reported series.

METHODS The authors conducted a retrospective review of data from consecutive patients with fusiform vertebrobasilar artery aneurysms treated with the PED.

RESULTS This review resulted in the identification of 12 such patients (mean [ \pm SD] age $55.1 \pm 14.1$ years). Eleven patients had symptoms; 1 had a dissecting aneurysm identified on imaging for neck pain. The average aneurysm size was $13.25 \pm 4.5 \mathrm{~mm}$. None of the aneurysms were ruptured or previously treated. The average clinical follow-up duration was $22.1 \pm 10.7$ months and radiological follow-up was $14.5 \pm 11.1$ months from the index PED treatment. One patient suffered a perforator stroke and had a modified Rankin Scale (mRS) score of 4 at last follow-up. Another patient had a retained stent pusher requiring retrieval via surgical cut-down but recovered to an mRS score of 0 at last follow-up. Eleven (91.7\%) of 12 patients recovered to an mRS score of 0 or 1 . Two patients had aneurysmal remnants at 7 and 10 months, respectively, after the index PED, which were retreated with PEDs. At last follow-up, all 12 aneurysms were occluded and PEDs were patent. The minimum follow-up duration was 12 months from the index PED treatment; no patient experienced delayed hemorrhage, stroke, or in-stent stenosis.

CONCLUSIONS Flow diversion with selective adjunctive techniques is evolving to become a safer treatment option for posterior circulation aneurysms. This is the longest clinical follow-up duration reported for a single-center experience of flow-diversion treatment of these aneurysms.

http://thejns.org/doi/abs/10.3171/2015.6.JNS1565

KEY WORDS flow diversion; fusiform vertebrobasilar aneurysm; Pipeline embolization device; vascular disorders

$\mathrm{F}$ Low diversion has proven to be an important addition to the endovascular toolbox for the treatment of anterior circulation intracranial aneurysms..$^{5,8,9,12,14,18}$ There has been considerable pessimism with respect to the use of flow diversion for posterior circulation aneurysms because of reports of perforator territory infarcts and de- layed ruptures, including an initial case series from our center. ${ }^{3-6,11,17}$ There have been recent reports of US singlecenter experiences ${ }^{1,13}$ and an Australian multicenter experience ${ }^{15}$ with posterior circulation flow diversion with acceptable morbidity and mortality rates $(0 \%-9.4 \%)$. In this paper we report 12 consecutive patients who underwent

ABBREVIATIONS AICA = anterior inferior cerebellar artery; $\mathrm{BA}=$ basilar artery; $\mathrm{CTA}=\mathrm{CT}$ angiography; $\mathrm{DSA}=$ digital subtraction angiography; $\mathrm{MRA}=\mathrm{MR}$ angiography; $\mathrm{mRS}=$ modified Rankin scale; $\mathrm{PCA}=$ posterior cerebral artery; $\mathrm{PED}=$ Pipeline Embolization Device; PICA = posterior inferior cerebellar artery; PRU = P2Y12 reactivity unit; $\mathrm{VA}=$ vertebral artery; $\mathrm{VB}=$ vertebrobasilar; $\mathrm{VBJ}=\mathrm{VB}$ junction.

SUBMITTED January 10, 2015. ACCEPTED June 18, 2015.

INCLUDE WHEN CITING Published online December 11, 2015; DOI: 10.3171/2015.6.JNS1565. 
flow diversion for large and giant vertebrobasilar (VB) artery aneurysms using strategies that were not previously applied and which resulted in markedly improved procedural safety and outcomes when compared with our previous series. ${ }^{17}$ In addition, we describe the results of longerterm radiological and clinical follow-up for these patients.

\section{Methods}

\section{Study Design, Setting, and Population}

After receiving institutional review board approval, a retrospective review of a prospectively collected database of endovascular patients was performed to identify consecutive patients with VB artery aneurysms who were treated with the Pipeline Embolization Device (PED; Covidien) between May 2011 and February 2014, subsequent to our previously reported series. ${ }^{17} \mathrm{~A}$ total of 12 patients were identified. The electronic charts of these patients were reviewed, and demographic and procedural data were analyzed. In each case, flow diversion was chosen for treatment after the exclusion of all other traditional endovascular (coiling, stent-assisted coiling) and microsurgical options (clip reconstruction or vessel sacrifice with or without bypass) during discussions at our weekly multidisciplinary peer-review conferences.

\section{Antiplatelet Regimen}

The patients in this series were started on a daily oral antiplatelet regimen consisting of aspirin $(325 \mathrm{mg})$ and clopidogrel $(75 \mathrm{mg})$ at least 7 days before the day of treatment. Platelet inhibition was tested routinely before the procedure with a goal response unit of 550 for aspirin and 200 for clopidogrel considered therapeutic levels (VerifyNow Assay System, Accumetrics, Inc.). If the aspirin response was subtherapeutic, a therapeutic response was achieved and confirmed by administering a loading dose of $650 \mathrm{mg}$ of aspirin before the procedure. If the response to clopidogrel was subtherapeutic, but the P2Y12 reactivity unit (PRU) value was close to 200, the patient was given an additional loading dose of $600 \mathrm{mg}$ of clopidogrel, and the assay was repeated. If the response was still subtherapeutic or PRU $>250$, the patient was switched to a 60 -mg loading dose of prasugrel followed by $5 \mathrm{mg}$ daily to replace the clopidogrel. Postprocedurally, patients were maintained on dual antiplatelet therapy for at least 6 months and aspirin for a lifetime.

\section{Procedural Details}

Patients in this series were treated with the PED. Procedures were performed with the patient under conscious sedation and after local infiltration of an anesthetic agent at the puncture site. Transfemoral access (unilateral or bilateral) was used in most cases. Brachial or radial artery access was used for cases with difficult femoral artery access. Access through both femoral arteries was used when the plan was to sacrifice 1 of the vertebral arteries (VAs) in cases in which there was flow from the other VA directly into the aneurysmal sac or a "jailed" catheter was required for adjunctive coil placement. After arterial access was obtained, $100 \mathrm{U} / \mathrm{kg}$ of heparin was administered to maintain an activated coagulation time of $280-350 \mathrm{sec}-$ onds throughout the duration of the procedure. The therapeutic effect of the heparin was allowed to wear off at the end of the procedure and was not reversed.

Coaxial-access flexible guide sheaths (Neuron MAX 088; Penumbra Inc.) and intermediate catheters (Navien 072 or Navien 058 , Covidien) were used to achieve intermediate catheter access (as a part of a triaxial access system) as close to the aneurysm as possible. This allowed better trackability of the PED and better sizing of the device to the target vessel. The best method to size the aneurysm and the parent vessel in our experience is to measure the size of the distal end of an intermediate catheter under high magnification in both anteroposterior and lateral planes to assess the magnification factor. The Marksman $150-\mathrm{cm}$ microcatheter (Covidien) was our standard microcatheter for delivery of the PED.

Adjunctive techniques primarily used coiling for a variety of purposes. Coiling was accomplished with a coiling catheter "jailed" in the aneurysm. The VA was sacrificed distal to the posterior inferior cerebellar artery (PICA) and any branches originating from the respective VA in cases where necessary, as described above.

\section{Follow-Up}

Posttreatment conventional digital subtraction angiography (DSA) was typically performed at 3-6 months. Patients who refused conventional angiography were offered the option of CT angiography (CTA) or MR angiography (MRA). If the initial CT or MR angiogram showed complete occlusion, a second imaging study was performed at 1-2 years via conventional angiography or either MRA or CTA, according to patient preference. Patients who experienced new neurological deficits underwent diffusion-weighted MRI to assess for ischemic events or a noncontrast head CT scan to assess for hemorrhage. All procedure-related complications were reported, regardless of clinical manifestation. Patients were considered to have procedure-related ischemic injury if restricted diffusion was demonstrated in any vascular distribution covered by the PED or PEDs. Clinical outcome was assessed according to the modified Rankin Scale (mRS) score. This score was obtained during a follow-up visit at our clinic or by telephone interview for patients referred from distant locales.

\section{Results}

\section{Descriptive and Outcome Data}

Twelve patients (Table 1) were treated with Pipeline flow diversion for fusiform posterior circulation aneurysms. The average age of these patients was $55.1 \pm 14.1$ years. Seven patients were men. Eleven patients were symptomatic, with headache $(\mathrm{n}=8)$, transient ischemic attack $(n=1)$, brainstem compression symptoms $(n=6)$, or cranial nerve palsies $(n=2)$. In 1 patient, the aneurysm was found incidentally during an evaluation for neck pain. The average aneurysm size was $13.25 \pm 4.5 \mathrm{~mm}$ ( 2 were $<$ $10 \mathrm{~mm}$, and 10 were $10-20 \mathrm{~mm}$ in maximum dimension). All 12 were fusiform in configuration. Six aneurysms were located at the VB junction (VBJ), 3 were VA aneurysms, 1 was a distal basilar artery (BA) aneurysm, 1 a mid-BA 


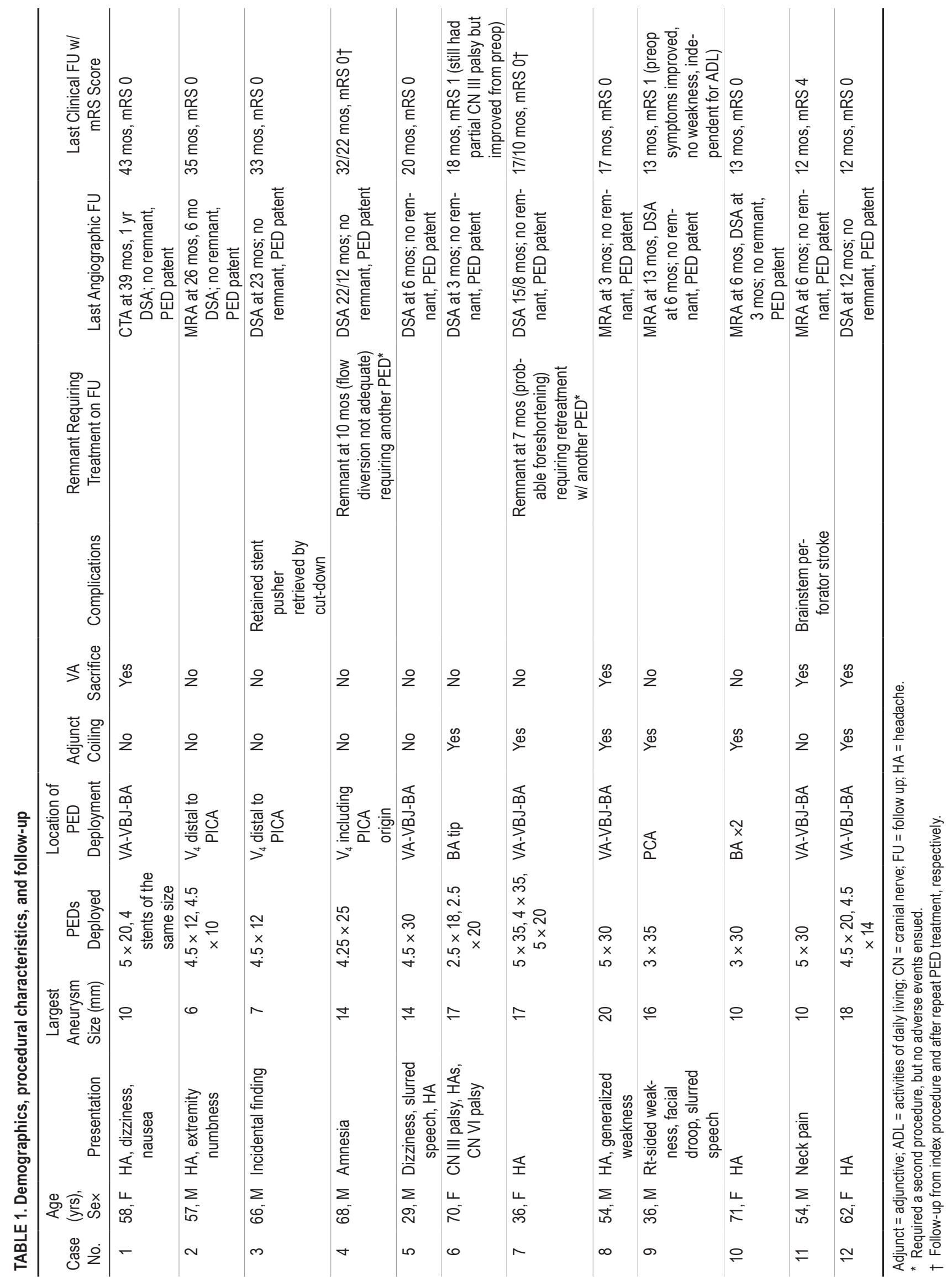


aneurysm, and 1 a posterior cerebral artery (PCA) aneurysm. None of the aneurysms had ruptured, and none of the patients had undergone previous treatment of the aneurysm. Transfemoral access was used in all cases except for 2 cases in which brachial or radial artery access was used, as in Case 10 (Fig. 1). Seven patients were treated with a single PED. Five patients had multiple devices to achieve good neck coverage as well as flow diversion. In all these cases, the overlap of the PEDs was in the aneurysmal segment and not in a normal BA segment or across critical branches, such as the anterior inferior cerebellar artery (AICA). Adjunctive coiling was performed in 6 patients, and VA sacrifice was performed in 4 .

\section{Complications}

There was a single case of technical difficulty in a patient (Case 3) with a VA aneurysm in whom the PED could not be opened distally and failed to separate from the pusher. We initially tried transecting the pusher at the radial artery access site and securing it there. However, the
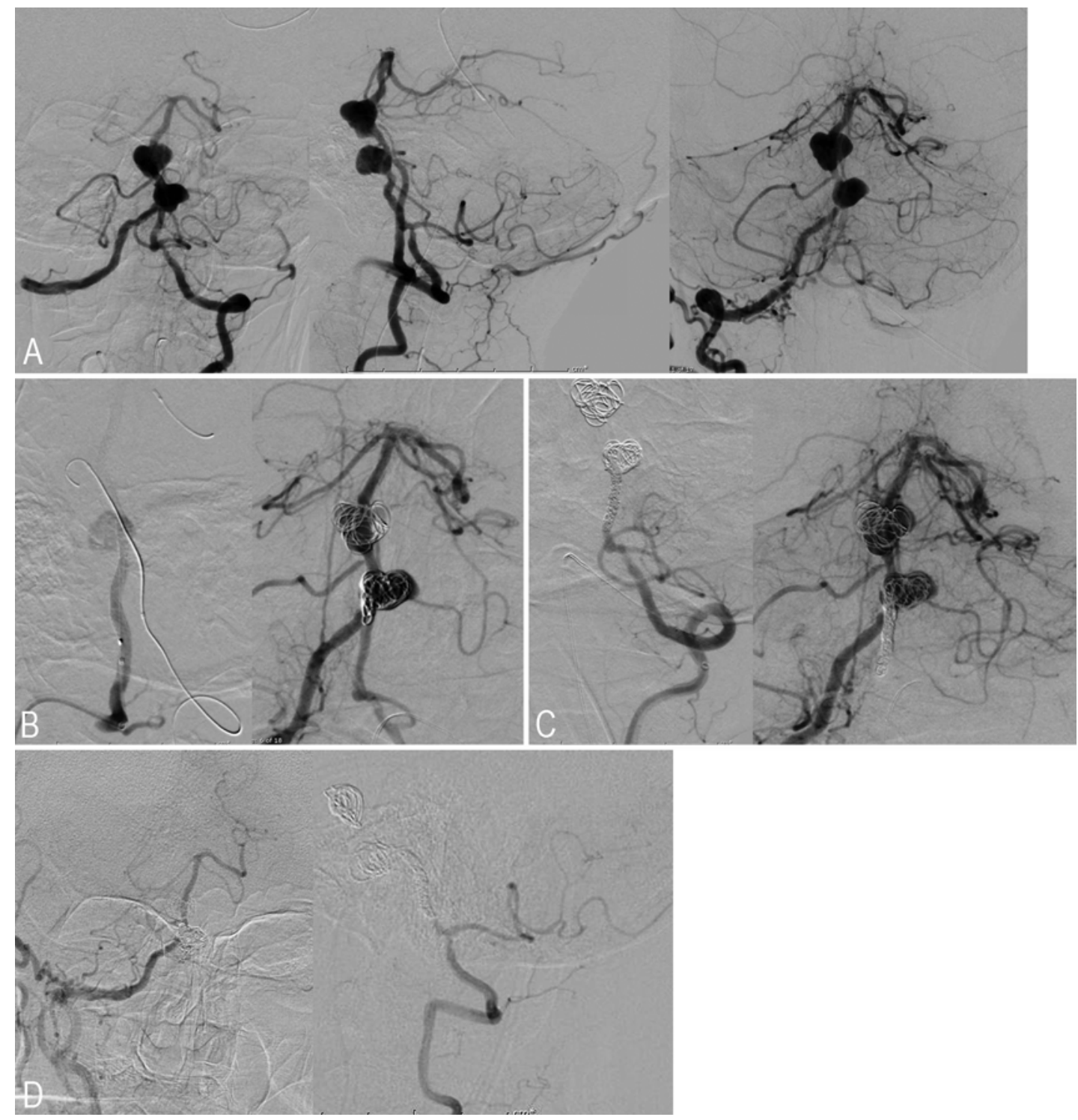

FIG. 1. Case 10. This patient had a history of headaches and significant vascular disease, including an aortic aneurysm and bilateral iliac stents, which precluded transfemoral access. Intermediate catheters were placed in both VAs using bilateral brachial access. A: Anteroposterior (left) and lateral (center) views from a left VA injection show VBJ and lower BA fusiform aneurysms. A right VA injection is also shown (right). B: A lateral view after right VA injection (left) shows 2 PEDs deployed from the PCA through the BA down to the right VA. There is a jailed coiling catheter in the left VA with a coil in the BA that is yet to be deployed. Anteroposterior view is shown after coils have been deployed in both aneurysms and the left VA sacrifice has begun (right). C: Completion of the left VA sacrifice distal to the PICA (left). Right VA injection after completion of left VA sacrifice (right). D: Anteroposterior view after right VA injection (left) and lateral view after left VA injection (right) 3 months after treatment showing complete obliteration of both aneurysms and patency of the posterior circulation. Reproduced with permission from Lin N, Brouillard AM, Krishna C, Mokin M, Natarajan SK, Sonig A, et al: Use of coils in conjunction with the Pipeline embolization device for treatment of intracranial aneurysms. Neurosurgery 76:142-149, 2015.7 
patient experienced radicular pain with any neck movement. A VA cut-down at the C-1 level was performed in a delayed fashion to transect the pusher wire there and retrieve the wire from the radial artery entry site. None of this was of any consequence, and the patient recovered to an mRS score of 0 and was stable at 33 months of followup. Another patient (Case 11) with a VBJ aneurysm who had PED placement along with coiling and VA sacrifice developed quadriparesis, dysphagia, and dysarthria (Fig. 2). A head CT scan did not show any hemorrhage, and an MR angiogram showed that the PED was patent. The patient was maintained on an eptifibatide infusion for 24 hours but did not improve. MRI showed a paramedian infarction in the upper medulla and lower pons, probably due to a perforator arising from the very distal VA that was occluded during vessel deconstruction. The patient eventually required a tracheostomy and percutaneous endoscopic gastrostomy, after which he was transferred to an inpatient skilled nursing facility with an mRS score of 4 . At the 12-month follow-up, this patient still had an mRS score of 4 , is able to ambulate with a walker, and has some difficulty swallowing liquids, but can take a pureed food diet. The tracheostomy has been closed.

\section{Follow-Up}

The average clinical follow up was $22.1 \pm 10.7$ months (range 12-43 months) from the index PED treatment; the average radiological follow-up was $14.5 \pm 11.1$ months (range 3-39 months). Two patients (16.6\%) had aneurysm remnants noted on follow-up angiography that required
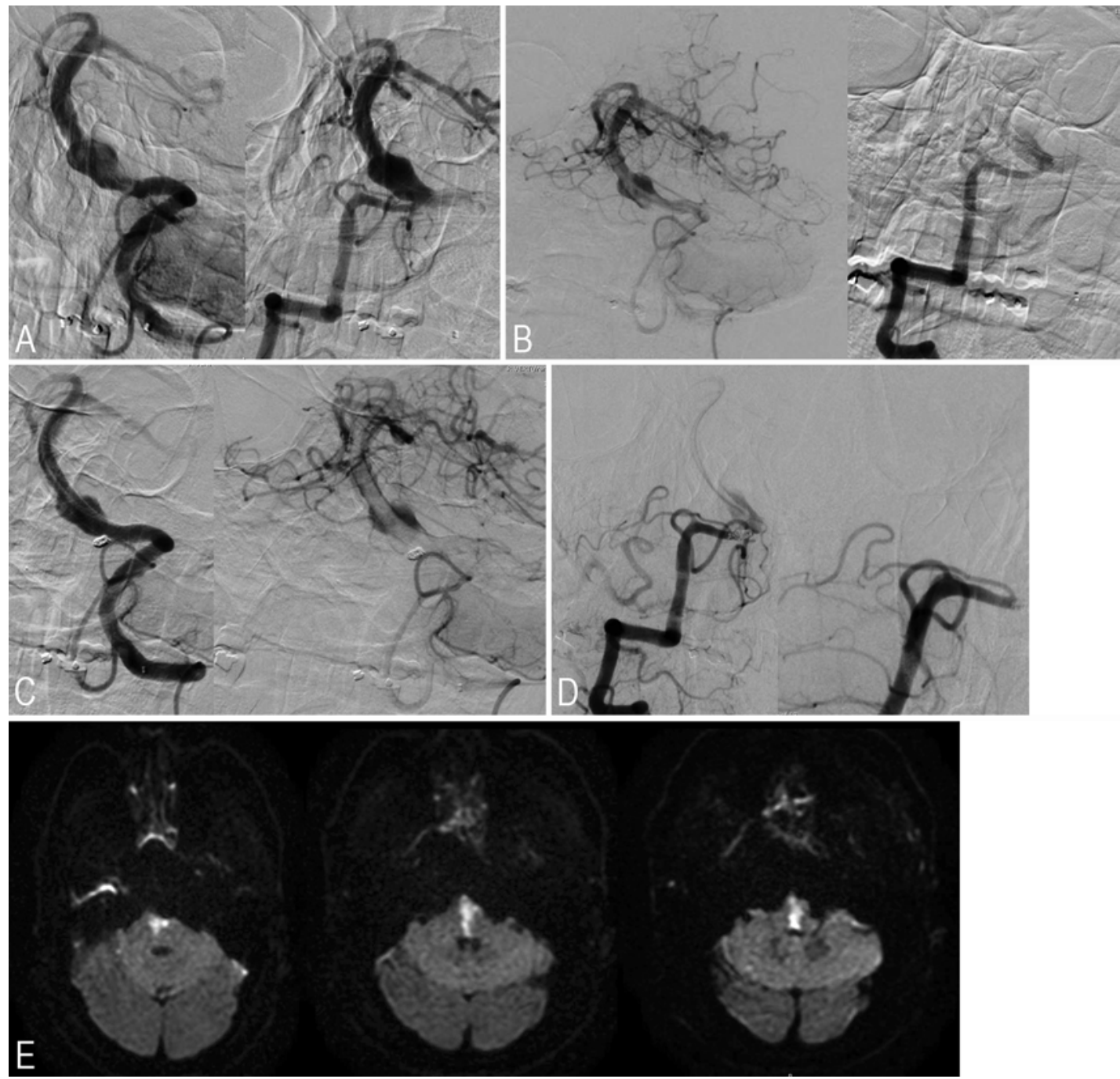

FIG. 2. Case 11. This patient was referred with a posterior circulation aneurysm that was increasing in size, as demonstrated by noninvasive imaging. The aneurysm was identified during evaluation of the patient's neck pain; the patient was neurologically intact at presentation. A: Left (left) and right (right) VA angiographic injection views show a fusiform aneurysm at the VBJ, involving the left and right VAs, with flow into the aneurysmal sac. B: Left VA injection after deployment of a single PED with some stasis of contrast flow in the aneurysm (left). Simultaneous right VA injection (right) shows the right VA filling the aneurysmal sac. C: Early arterial phase (left) and late arterial phase (right), after placement of a few coils in the right VA for sacrifice, showing more stasis of contrast material in the aneurysm. D: Further coiling for right VA sacrifice shows occlusion of small arterial branches at the VBJ (left, before the last coil; right, after the last coil, showing occlusion of perforators). E: Consecutive slices acquired via diffusionweighted MRI show brainstem infarct. 
retreatment: Case 4 did not have adequate flow diversion as noted on the 7-month follow-up angiogram, and Case 7 had a foreshortening of the PED and aneurysm filling at 10 months. Both patients underwent PED retreatment, and no aneurysm remnants were observed on follow-up angiograms at 12 and 8 months after retreatment, respectively. The remaining 10 patients had patent parent vessels with no aneurysm remnants and no in-stent stenosis of the PED at the last angiographic follow-up evaluation. There were no delayed hemorrhagic or ischemic events or PED-related complications in this series.

\section{Discussion}

Posterior circulation fusiform aneurysms have an ominous natural history if left untreated, with a reported mortality rate of 30\%.,16 Favorable overall long-term outcome was achieved in $90 \%$ of patients with PCA aneurysms but only in $60 \%$ of those with VA-PICA aneurysms and $39 \%$ of those with BA-VBJ aneurysms treated with traditional endovascular and surgical techniques. ${ }^{2}$ We previously reported on 6 patients who underwent PED flow-diversion treatment: 4 aneurysms (66\%) were fusiform holobasilar, 1 was fusiform midbasilar, and the last was a fusiform distal BA trunk. ${ }^{17}$ Five $(83.3 \%)$ of 6 patients in this series presented with ischemic symptoms, and $3(50 \%)$ had pretreatment strokes as demonstrated by MRI. An average of $5.3 \pm 2.9$ devices (range 3-9 devices) were used in these 6 patients, and 5 of them experienced posttreatment brainstem ischemic events. None of these patients underwent adjunctive coiling of the aneurysmal sac. One aneurysm ruptured 24-48 hours after PED placement and another at 8 weeks after initial treatment. During the follow-up period, 4 patients $(67 \%)$ died, 1 was disabled (mRS score of $5)$, and 1 recovered to $\mathrm{mRS}$ score of 0 .

In the current series, 10 of 12 patients had uneventful PED placement and were discharged home without any technical or clinical consequences. One patient had a retained pusher that required delayed surgical retrieval but did not have any disabling consequences, and 1 patient suffered a disabling perforator stroke. There were no deaths. These results are markedly better than those reported in our previous publication. ${ }^{17}$ We attribute these improved results to the following factors.

\section{Patient Selection}

The first factor we believe to improve results is better patient selection. Three patients had VA-PICA aneurysms, 6 had VBJ aneurysms, 2 had BA tip aneurysms, and 1 had a PCA aneurysm. There were no fusiform holobasilar aneurysms in this series. Only 3 patients $(25 \%)$ in this series presented with ischemic symptoms, and none had evidence of strokes on pretreatment MRI. Thus, the patients who presented in this series presented earlier when compared with patients reported in the previous series. Patients presenting with acute ischemic symptoms from brainstem infarction and compression did not do well in our previous series. Although flow diversion was performed uneventfully, these patients progressed to have brainstem strokes that resulted in disability or death. This may be reflective of already compromised ostia of affected perforators as well as the large number of devices as noted below used to treat the aneurysm. Aneurysms in perforator-rich regions, especially fusiform mid-BA aneurysms, have a higher chance of perforator infarcts after flow diversion. Patients with partially thrombosed aneurysms with significant clot burden also have a higher risk of perforator strokes. We do not believe flow diversion in its current iteration is an option for giant holobasilar fusiform dolichoectatic aneurysms with major branch points coming off opposite walls of the aneurysm (Fig. 3). Typically, these aneurysms have layers of thrombi along their walls and tenuous patency of perforators through channels within the thrombus.

\section{Antiplatelet Regimen}

The second factor may have been critical attention to the antiplatelet regimen. We were very strict with respect to confirming the therapeutic effect of antiplatelet therapy by using aspirin and clopidogrel response testing before flow diversion (as mentioned above), and we believe that has significantly reduced the number of ischemic complications overall in our series.

\section{Number of PEDs}

The third factor we believe was limiting the number

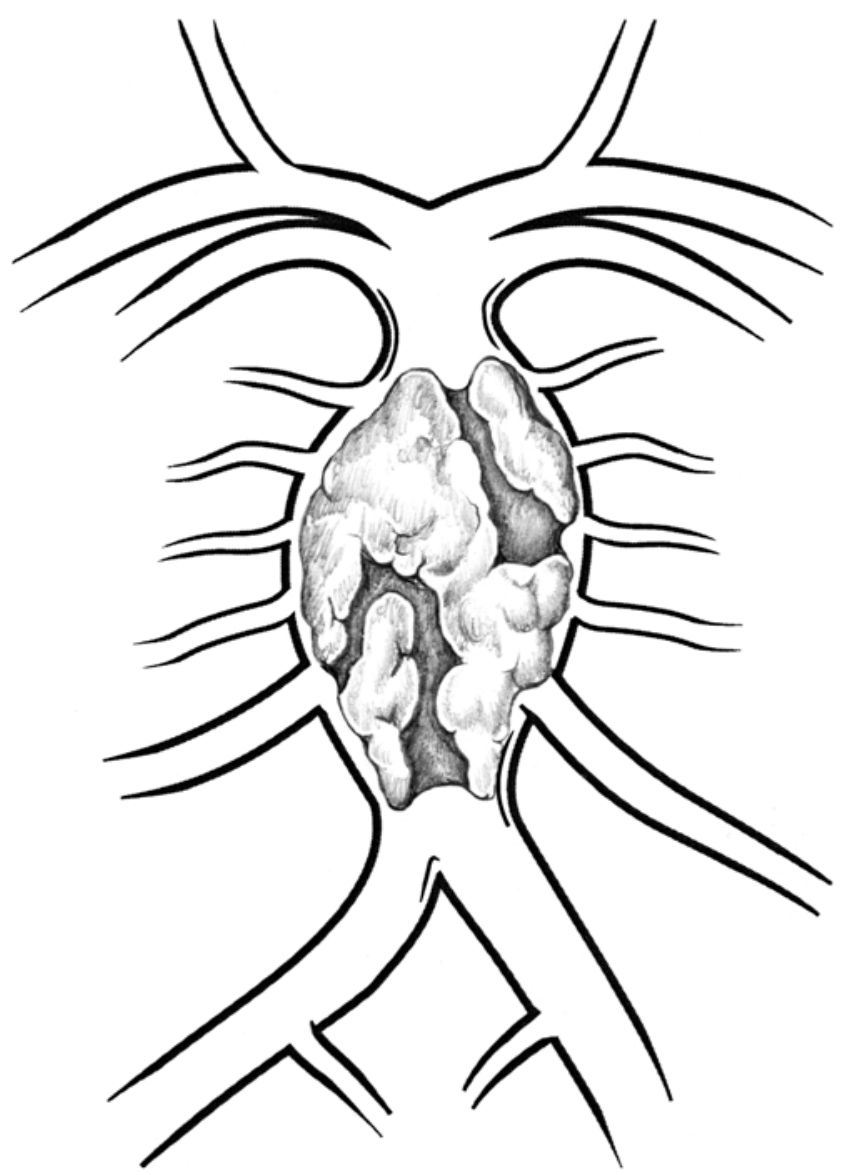

FIG. 3. Illustration showing a fusiform thrombosed holobasilar aneurysm with multiple patent branches on the walls of the aneurysm with preserved flow in them through the thrombus. These aneurysms are not good candidates for flow diversion and carry a high risk of brainstem stroke. Copyright University at Buffalo Neurosurgery. Published with permission. 
of PEDs. In this series, only 5 patients had multiple PEDs (average $1.67 \pm 0.98$ PEDs, range 1-4) used for flow diversion. This is in sharp contrast to the number of PEDs used in aneurysms in the previous series and probably decreased the number of posttreatment ischemic strokes in the present series. The previous series was reported when the longest devices were $20 \mathrm{~mm}$ long, however now we routinely have $35-\mathrm{mm}$ devices that allow for easier construction of longer PED constructs. Whenever possible, we cover the aneurysm neck with a single PED. We use a second device after deployment of the first solely to ensure adequate coverage of the aneurysm neck. This is mostly in the case of giant aneurysms where 1 device is not long enough to provide adequate coverage of the entire aneurysm and a long-enough anchor segment in the normal segments proximal and distal to the aneurysm. We do not use a second device to achieve aneurysm stasis or better flow diversion. This is of critical importance, because we have previously demonstrated that a single device when packed can result in greater than $50 \%$ metal surface coverage, and 2 devices seriously threaten the patency of the critical perforators that are typically crowded around the necks of these aneurysms. ${ }^{10}$ By using a single device over perforator segments, we have significantly reduced the occurrence of large, devastating perforator strokes.

\section{Adjunctive Coiling}

Another factor may be adjunctive coiling. Coiling was performed in 6 patients in this series and has become routine for cases in which coils can be accommodated in the aneurysmal sac.

We believe that the coils reduce stent prolapse in the fusiform component of the aneurysm by acting as a scaffold for the PED.7 Typically, braided flow diverters such as the PED foreshorten significantly during and often even after deployment. Because fusiform aneurysms are larger than the largest diameter of these devices and involve long segments of affected vessels, the tendency to foreshorten is increased. By deploying coils within the most saccular segment of the fusiform aneurysm, the PED is fixed in place and is less likely to shift or foreshorten.

Using coils in the most saccular component of the aneurysm, particularly in large or giant aneurysms, serves as a scaffold to organize thrombi. Coils provide a second layer of protection from hemorrhagic complications by altering intraaneurysmal flow dynamics and allowing controlled intraaneurysmal thrombosis and eventual reorganization into stable fibrous tissue. This alteration of thrombus organization prevents large thrombolytic cleavage planes to occur within and around a large clot, as can occur during inflammatory reorganization within the thrombus hours to days and weeks after treatment. It is unclear how many coils are needed to provide this function; we believe the packing density needs to be less than with stent- or balloon-assisted or primary coiling but more than simply 1 or 2 coils. We have begun some studies using computational fluid dynamics to assess the hemodynamic value of adjunctive coiling with flow diversion, and the most effective coil mass by volume appears to be $11 \%$ (J. Xiang et al., personal communication, 2014).

The third application of coils is to help appose the PED to key perforators, especially the AICA, by placing a single or a few coils along the opposite wall through a jailed microcatheter during deployment of the PED. We believe that preservation of the AICA is a crucial part of preventing major ischemic injury.

These are our hypotheses to explain the achievement of better results in this series; however, they need to be proven in larger prospective multicenter studies with control subjects.

We encountered 1 case of a VBJ aneurysm in which a perforator infarction ensued, and the patient was disabled with an mRS score of 4 at follow-up. The patient was intact immediately after the procedure but developed symptoms 30 minutes later that slowly progressed over the next 24 hours. The patient has displayed marked functional improvement but remains dependent for activities of daily living. An MRI study obtained at 24 hours showed a medial infarct at the pontomedullary junction corresponding to the short midline perforators from the lower BA to the VBJ. A retrospective review of the angiogram also revealed occlusion of a small perforator from the distal VA that occurred during contralateral VA sacrifice. This case emphasizes the need to pay close attention to even small branches that arise near the aneurysm when performing these procedures. There is no way to reliably predict whether an individual patient will have complications because of perforator occlusion.

Table 2 summarizes published reports of the limited experience with Pipeline flow diversion for posterior circulation aneurysms..$^{1,13,15,17,19}$ After our initial report of 7 patients with fusiform posterior circulation aneurysms, ${ }^{17}$ Phillips et

TABLE 2. Reports of flow diversion for posterior circulation aneurysms

\begin{tabular}{|c|c|c|c|c|c|c|c|}
\hline Authors \& Year & No. of Patients & $\begin{array}{l}\text { No. of Fusiform } \\
\text { Aneurysms }\end{array}$ & $\begin{array}{c}\text { No. of Ischemic } \\
\text { Complications (\%) }\end{array}$ & $\begin{array}{l}\text { No. of Hemorrhagic } \\
\text { Complications(\%) }\end{array}$ & $\begin{array}{c}\text { No. of Disabilities } \\
\text { Related to PEDs } \\
(\%)\end{array}$ & $\begin{array}{l}\text { No. of Deaths } \\
\text { Related to } \\
\text { PEDs (\%) }\end{array}$ & $\begin{array}{c}\text { Mean FU } \\
\text { (mos) }\end{array}$ \\
\hline $\begin{array}{l}\text { Phillips et al., } 2012 \\
\text { ( } 3 \text { centers })\end{array}$ & 32 & 20 & $3(9.4)$ & $2(6.3)$ & $3(9.4)$ & 0 & 21 \\
\hline Siddiqui et al., 2012 & 7 & 3 & $5(71.4)$ & $2(28.6)$ & $1(14.3)$ & $2(28.6)$ & 4.5 \\
\hline Chalouhi et al., 2013 & 7 & 2 & 0 & 0 & 0 & 0 & 5 \\
\hline Toth et al., 2015 & 6 (7 aneurysms) & 2 & $3(50)$ & 0 & $2(33)$ & $1(16.6)$ & 14.5 \\
\hline Munich et al., 2014 & 12 & NR & $4(33)$ & 0 & $3(25)$ & $1(8.3)$ & 11 \\
\hline Present series, 2014 & 12 & 12 & $1(8.3)$ & 0 & $1(8.3)$ & 0 & 22 \\
\hline
\end{tabular}

$\mathrm{NR}=$ not reported. 
al. ${ }^{15}$ reported their experience with flow diversion for the treatment of 32 posterior circulation aneurysms, including 20 of fusiform morphology, at 3 centers in Australia. They did not report the fusiform aneurysm results separately. Among the 32 posterior circulation aneurysm treated in this series, including 8 patients with ruptured aneurysms, perforator territory infarctions occurred in $3(14 \%)$ of the 21 patients with BA aneurysms, and in all 3, a single PED was used. Two asymptomatic intracranial hematomas were recorded. No aneurysmal rupture or PED thrombosis was encountered. The overall rate of permanent neurological complications was $9.4 \%$ (3/32); all 3 patients had very mild residual symptoms and a good clinical outcome. The mean follow-up duration was 21 months. Aneurysm occlusion was demonstrated in $85 \%$ of patients with more than 6 months of follow-up and $96 \%$ of patients with more than 1 year of follow-up. No delayed parent vessel narrowing or hemorrhagic complications occurred. Chalouhi et al. ${ }^{1}$ reported 2 patients with fusiform aneurysms who were treated by flow diversion. One aneurysm was a VA aneurysm and the other was a BA aneurysm. Only 1 PED was used in each patient. Adjunctive coiling was not used in either patient. The average clinical follow-up duration was 5 months, and no ischemic complications, death, or disability related to flow diversion were encountered. Toth et al..$^{19}$ reported their results with flow diversion treatment of 2 fusiform aneurysms. Both were midbasilar aneurysms. An average of 3.5 devices was used. Adjunctive coiling was not used. Both patients had ischemic complications, one immediately and the other after 4.5 months. Both patients were disabled with mRS scores of 4 and 5 , respectively, after the ischemic event; and the first patient died approximately 18 months after the procedure. Munich et al..$^{13}$ reported the results of 12 posterior circulation aneurysms treated by flow diversion, of which 2 had ruptured. No mention was made of the number of fusiform aneurysms nor the specific locations of these aneurysms within the posterior circulation. A mean of 3.25 devices was used per patient. Adjunctive coiling was not used. The average clinical follow-up duration was 11 months and the average radiological follow-up was 4.9 months. Four patients (33\%) had ischemic complications; 2 of them were disabled and 1 other died due to disability. The high rate of ischemic complications in these patients when compared with our current series may be related to the number of devices that were deployed in these patients, as mentioned previously. There were no delayed hemorrhages in their series.

Our current series of 12 patients is the largest series of unruptured fusiform aneurysms reported from a single center. Our mean clinical follow-up of 22 months is the longest reported for these patients, and every patient had at least 12 months of follow-up. Only 1 patient had an ischemic stroke and is disabled as a result, and this was clearly related to sacrifice of brainstem perforators during VA sacrifice. The remaining $91.7 \%$ of patients had favorable outcomes (at last follow-up, 9 with an mRS score of 0,2 with mRS scores of 1). These are very good results compared with the natural history of the disease and the results associated with conventional microsurgical and endovascular techniques. There were no delayed hemorrhages or ischemic complications in our series, and we think this could be related to the low number of devices used per patient and the use of adjunctive coiling. Two patients needed repeat flow diversion after 7 and 10 months to achieve aneurysm occlusion, but these procedures were performed without consequence. Although our series has the inherent limitations of a small case series from a single center, the results of the current consecutive series of patients are dramatically different from our previously reported results. This series also provides insight into patient selection and procedural nuances to avoid complications and achieve good outcomes in these patients with an ominous natural history and poor outcomes after traditional techniques.

\section{Generalizability}

Careful patient selection and attention to perioperative management and technical nuances can reduce the number of patients with ischemic or hemorrhagic complications, making flow diversion a viable treatment option for these patients given the grim natural history of these aneurysms and the limited treatment options available. ${ }^{2}$ Moreover, improvement in flow-diversion device technology and an understanding of the flow dynamics and pathophysiology of aneurysm occlusion after flow diversion will make these procedures safer and associated with fewer complications than are reported in current series.

\section{Limitations}

This is a retrospective review of a small number of patients treated by flow diversion and has inherent limitations due to the study design. During the same period, 22 patients with posterior circulation aneurysms were managed with simple coiling $(\mathrm{n}=10)$ or stent-assisted coiling $(\mathrm{n}=12)$ at our center. Given the limited number of patients with posterior circulation aneurysms, the variability of anatomy and technical nuances, operator preferences involved in managing each individual case, and the retrospective nature of this report, drawing a meaningful comparison between patients treated with and without PEDs is difficult. Although the results are comparable to a few recent published reports, other recent reports do not provide detailed insight on patient selection and how to avoid complications, and our follow-up is the longest ever reported for patients with posterior circulation aneurysms treated by flow diversion. The conclusions of this paper represent the evolving opinions of our team that arose from our experience with the treatment of these patients and are not a reflection of validated scientific proof. Once the experience and the learning curve associated with the treatment of these patients with this technology and the evolution of flow diversion technology stabilize across multiple centers, a study that validates Pipeline embolization for these patients may be feasible.

\section{Conclusions}

At our center, flow diversion is evolving to become a safer treatment option for posterior circulation aneurysms that are not amenable to treatment with traditional techniques. Outcomes after flow diversion are better than the natural history of the disease and those obtained by traditional techniques, with adherence to diligent patient selection and procedural and periprocedural nuances. 


\section{Acknowledgments}

We thank Paul H. Dressel, BFA, for preparation of the illustrations, and Debra J. Zimmer for editorial assistance.

\section{References}

1. Chalouhi N, Tjoumakaris S, Dumont AS, Gonzalez LF, Randazzo C, Starke RM, et al: Treatment of posterior circulation aneurysms with the pipeline embolization device. Neurosurgery 72:883-889, 2013

2. Coert BA, Chang SD, Do HM, Marks MP, Steinberg GK: Surgical and endovascular management of symptomatic posterior circulation fusiform aneurysms. J Neurosurg 106:855-865, 2007

3. de Barros Faria M, Castro RN, Lundquist J, Scrivano E, Ceratto R, Ferrario A, et al: The role of the pipeline embolization device for the treatment of dissecting intracranial aneurysms. AJNR Am J Neuroradiol 32:2192-2195, 2011

4. Fiorella D, Hsu D, Woo HH, Tarr RW, Nelson PK: Very late thrombosis of a Pipeline embolization device construct: case report. Neurosurgery 67 (3 Suppl Operative):onsE313onsE314, 2010

5. Fischer S, Vajda Z, Aguilar Perez M, Schmid E, Hopf N, Bäzner H, et al: Pipeline embolization device (PED) for neurovascular reconstruction: initial experience in the treatment of 101 intracranial aneurysms and dissections. Neuroradiology 54:369-382, 2012

6. Klisch J, Turk A, Turner R, Woo HH, Fiorella D: Very late thrombosis of flow-diverting constructs after the treatment of large fusiform posterior circulation aneurysms. AJNR Am J Neuroradiol 32:627-632, 2011

7. Lin N, Brouillard AM, Krishna C, Mokin M, Natarajan SK, Sonig A, et al: Use of coils in conjunction with the Pipeline embolization device for treatment of intracranial aneurysms. Neurosurgery 76:142-149, 2015

8. Lubicz B, Collignon L, Raphaeli G, De Witte O: Pipeline flow-diverter stent for endovascular treatment of intracranial aneurysms: preliminary experience in 20 patients with 27 aneurysms. World Neurosurg 76:114-119, 2011

9. Lylyk P, Miranda C, Ceratto R, Ferrario A, Scrivano E, Luna $\mathrm{HR}$, et al: Curative endovascular reconstruction of cerebral aneurysms with the pipeline embolization device: the Buenos Aires experience. Neurosurgery 64:632-643, N6, 2009

10. Ma D, Xiang J, Choi H, Dumont TM, Natarajan SK, Siddiqui $\mathrm{AH}$, et al: Enhanced aneurysmal flow diversion using a dynamic push-pull technique: an experimental and modeling study. AJNR Am J Neuroradiol 35:1779-1785, 2014

11. Martin AR, Cruz JP, Matouk CC, Spears J, Marotta TR: The pipeline flow-diverting stent for exclusion of ruptured intracranial aneurysms with difficult morphologies. Neurosurgery 70 (1 Suppl Operative):21-28, 2012

12. McAuliffe W, Wycoco V, Rice H, Phatouros C, Singh TJ, Wenderoth J: Immediate and midterm results following treatment of unruptured intracranial aneurysms with the pipeline embolization device. AJNR Am J Neuroradiol 33:164-170, 2012

13. Munich SA, Tan LA, Keigher KM, Chen M, Moftakhar R, Lopes DK: The Pipeline Embolization Device for the treatment of posterior circulation fusiform aneurysms: lessons learned at a single institution. J Neurosurg 121:1077-1084, 2014

14. Nelson PK, Lylyk P, Szikora I, Wetzel SG, Wanke I, Fiorella D: The pipeline embolization device for the intracranial treatment of aneurysms trial. AJNR Am J Neuroradiol 32:34-40, 2011

15. Phillips TJ, Wenderoth JD, Phatouros CC, Rice H, Singh TP, Devilliers L, et al: Safety of the pipeline embolization device in treatment of posterior circulation aneurysms. AJNR Am J Neuroradiol 33:1225-1231, 2012

16. Raphaeli G, Collignon L, De Witte O, Lubicz B: Endovascular treatment of posterior circulation fusiform aneurysms: single-center experience in 31 patients. Neurosurgery 69:274-283, 2011

17. Siddiqui AH, Abla AA, Kan P, Dumont TM, Jahshan S, Britz GW, et al: Panacea or problem: flow diverters in the treatment of symptomatic large or giant fusiform vertebrobasilar aneurysms. J Neurosurg 116:1258-1266, 2012

18. Szikora I, Berentei Z, Kulcsar Z, Marosfoi M, Vajda ZS, Lee $\mathrm{W}$, et al: Treatment of intracranial aneurysms by functional reconstruction of the parent artery: the Budapest experience with the pipeline embolization device. AJNR Am J Neuroradiol 31:1139-1147, 2010

19. Toth G, Bain M, Hussain M, Moskowitz S, Masaryk T, Rasmussen P, et al: Posterior circulation flow diversion: a singlecenter experience and literature review. J Neurointerv Surg 7:574-583, 2015

\section{Disclosures}

Dr. Levy is a shareholder and/or has ownership interests in Intratech Medical Ltd., Blockade Medical LLC, and Medina Medical; is a principal investigator for Covidien US SWIFT PRIME trials; has received honoraria for training and lecturing from Covidien; has served as a consultant to Pulsar, Medina Medical, and Blockade Medical; and has received other financial support from Abbott for carotid training for physicians. Dr. Rai has served as a consultant to Stryker Neurovascular and Codman Shurtleff. Dr. Siddiqui has received research grants (not related to the present study) from the NIH and the University at Buffalo (Research Development Award); has financial interests in Hotspur, Intratech Medical, StimSox, Valor Medical, Blockade Medical, and Lazarus Effect; has served as a consultant to Codman \& Shurtleff Inc., Concentric Medical, Covidien Vascular Therapies, GuidePoint Global Consulting, Penumbra, Stryker Neurovascular, and Pulsar Vascular; has served on speakers' bureaus for Codman \& Shurtleff and Genentech; is on National Steering Committees for the Penumbra 3D Separator Trial, Covidien SWIFT PRIME Trial, and MicroVention FRED Trial; serves on the advisory board for Codman \& Shurtleff and Covidien Vascular Therapies; and has received honoraria from Abbott Vascular and Codman \& Shurtleff, Inc., for training other neurointerventionists in carotid stenting and for training physicians in endovascular stenting for aneurysms.

\section{Author Contributions}

Conception and design: Siddiqui, Natarajan, Rai, Carpenter, Levy. Acquisition of data: all authors. Analysis and interpretation of data: all authors. Drafting the article: Natarajan. Critically revising the article: all authors. Reviewed submitted version of manuscript: all authors. Statistical analysis: Natarajan.

\section{Correspondence}

Adnan H. Siddiqui, Department of Neurosurgery, University at Buffalo, 100 High St., Ste. B4, Buffalo, NY 14203. email: asiddiqui@ubns.com. 